TITLE:

\title{
Predicting Voltage Instability of Power System via Hybrid System Reachability Analysis
}

$\operatorname{AUTHOR}(S)$ :

Susuki, Yoshihiko; Hikihara, Takashi

\section{CITATION:}

Susuki, Yoshihiko ... [et al]. Predicting Voltage Instability of Power System via Hybrid System Reachability Analysis. Proceedings of the American Control Conference 2007: 4166-4171

ISSUE DATE:

2007-07

URL:

http://hdl.handle.net/2433/73437

RIGHT:

(C)2007 IEEE. 


\title{
Predicting Voltage Instability of Power System via Hybrid System Reachability Analysis
}

\author{
Yoshihiko Susuki and Takashi Hikihara
}

\begin{abstract}
Estimating voltage instability of power systems is important for reduction of large blackouts. This paper proposes to use reachability analysis of hybrid systems for predicting voltage instability of a power system. The reachability analysis is performed by computing backward reachable sets for unsafe sets of hybrid automata. The automata represent continuous voltage dynamics and discrete operations by relay devices. The unsafe sets of hybrid automata are also subsets of state space in which a system voltage shows unacceptable levels such as low or high values. With single machine-load bus (SMLB) system with controlled route switching, we show that voltage instability in the SMLB system can be predicted using the reachability analysis. The obtained result implies that voltage instability leading to large blackouts is possibly detected before its occurrence in power systems.
\end{abstract}

\section{INTRODUCTION}

The problem of estimating voltage instability is of paramount importance for power system operation and planning. Voltage instability is concerned with a power system's ability to maintain acceptable levels of voltages, following a load variation or event disturbance [1]. The instability implies in this paper that a power system is under unacceptable levels of voltages. There were many power system blackouts caused by voltage instability, including Japan and Sweden [2], and North Europe [3]. Recent regulatory reforms and uses of distributed power sources make it more difficult to keep safe levels of system voltages. It is by now widely recognized that such voltage dynamics cannot be fully estimated using conventional methods. A comprehensive approach has been therefore strongly required.

Hybrid systems theory has potential to provide a novel framework of voltage instability estimation of power systems. Hybrid dynamical systems and their control are active research subjects in computer science and control engineering [4]. Several researchers have recently worked on hybrid systems theory and voltage stability problems. Hiskens et al. [5], [6] propose a hybrid modeling of power systems including transformer tap changes and relay operations. Tong et al. [7] propose a hybrid system view of voltage instability problem. Geyer et al. [8], [9] and Leirens et al. [10] use hybrid control theory for voltage stability enhancement.

This paper proposes a numerical method for predicting voltage instability of a power system based on hybrid systems theory. The term prediction here implies that we determine voltage instability in a power system using numerical simulations of the corresponding dynamical model before its occurrence. A hybrid system-based approach to modeling and stability analysis of power systems is proposed in [11],

This research is supported in part by the Ministry of Education, Culture, Sports, Sciences and Technology in Japan, The 21st Century COE Program \#14213201 and Young Research (B) \#18760216, 2006.

The authors are with the Department of Electrical Engineering at Kyoto University, Katsura, Nishikyo, Kyoto, 615-8510, Japan (susuki@dove.kuee.kyoto-u.ac.jp; hikihara@kuee.kyoto-u.ac.jp).
[12]. The modeling is performed via hybrid automata [13] The stability analysis is based on reachability analysis of hybrid automata which is a well-established technique of safety verification in engineering systems [14]. This paper focuses on voltage instability following an event disturbance, called transient voltage instability [1], and proposes a numerical method for predicting the voltage instability. The method is demonstrated via an analysis of single machineload bus (SMLB) system. We show that transient voltage instability in the SMLB system can be predicted using the reachability analysis. A similar approach to transient angle instability is reported in [15]. The approach requires a different mathematical formulation from that in this paper.

\section{Predicting Voltage instability Via REACHABILITY ANALYSIS: A PROPOSED METHOD}

The second section introduces a numerical method for predicting the voltage instability. It is based on modeling of voltage dynamics via hybrid automata and reachability analysis of the hybrid automata.

\section{A. Definition of hybrid automaton as power system model}

A hybrid automaton $H$ [14] is defined to be a collection

$$
H=(Q \cup X, \text { Init, In, } f, \text { Dom, } e),
$$

where

- $Q \cup X$ is the union of discrete and continuous states. The state of $H$ is represented as a pair $(q, x)$, describing the discrete and continuous state;

- Init $\subseteq(Q \cup X)$ is a set of initial states;

- In $=(\mathcal{U} \cup \mathcal{D}) \cup\left(\Sigma_{u} \cup \Sigma_{d}\right)$ is the union of actions and inputs. $u \in \mathcal{U}$ is used to represent variables that can be controlled, called control inputs, and $d \in \mathcal{D}$ represents disturbance inputs, which are variables that cannot be controlled. $\sigma_{u} \in \Sigma_{u}$ represents control actions and $\sigma_{d} \in$ $\Sigma_{d}$ disturbance actions;

- $f$ is a function that takes state and input and maps to a new state $f:(Q \cup X) \times \operatorname{In} \rightarrow(Q \cup X)$. $f$ represents a continuous vector field or continuous-state control system indexed by the discrete state;

- Dom is called a domain and describes, for each discrete state, the subset of the continuous state space within which the continuous state may exist;

- $e:(Q \cup X) \times \operatorname{In} \rightarrow 2^{Q \cup X}$ is a transition relation and describes the transition logic, which may depend on continuous state and input, as well as discrete state and action.

Trajectories of the hybrid automaton $H$ evolve continuously as well as in discrete jumps. Mathematical descriptions of its trajectories and behavior are presented in [14] and references therein. 
The hybrid automaton $H$ can combine continuous voltage dynamics with discrete operations of power systems. The vector field $f$ in $H$ describes continuous voltage dynamics of generators and loads, and dynamic response of control systems. The discrete variables $\{q\}$ are assigned to system configurations or modes. A conventional setting [1] of stability study implies that there are three discrete variables assigned to pre-fault, fault-on, and post-fault modes. Transformer tap positions and shunt capacitor states should be also represented by the discrete variables for voltage instability estimation. The transition relation $e$ can then describe topological changes of transmission networks and changes of transformer tap positions and shunt capacitor states. The transition $e$ is driven by control and disturbance actions $\left(\sigma_{u}[\cdot], \sigma_{d}[\cdot]\right) \in\left(\Sigma_{u} \cup \Sigma_{d}\right)$. The actions include controlled and uncontrolled operations of relay devices, transformer tap, and shunt capacitors, and unanticipated event disturbances such as lightning and timber contact. The hybrid automaton $H$ can also comprise continuous state controllers such as dc links [16] and SVCs (Static Var Compensators) [17] by $u(\cdot) \in \mathcal{U}$ and include unregulated power flow due to electricity trading by $d(\cdot) \in \mathcal{D}$. The hybrid automaton $H$ is hence applicable to modeling of voltage dynamics with considering discrete operations.

\section{B. Predicting voltage instability via reachability analysis of hybrid automaton}

The present subsection introduces a numerical method for predicting the voltage instability. This prediction is based on reachability analysis of hybrid automata [14]. Now define an unsafe set $G \subset(Q \cup X)$ for the hybrid automaton $H$. This unsafe set is interpreted as a set of operating conditions in which a system voltage shows unacceptable levels such very high or low values. A backward reachable set $R_{t}(G)$ for the time $t(<0)$ in the hybrid automaton $H$ is then defined by a subset of $Q \cup X$ in which any state reaches the boundary $\partial G$ of $G$ in time 0 to $|t|$ despite of any control $\left(u(\cdot), \sigma_{u}[\cdot]\right)$. The term despite of any control implies that $G$ is reachable despite controls acting to avoid $G$. A mathematical description of the backward reachable set is given in [14] and references therein. The reachable sets are important for estimating the voltage instability. If a state exists in $R_{t}(G)$, then it can be evaluated that the system voltage will reach the unacceptable levels in time 0 to $|t|$. This estimation is possible at any time in operations such as onset of fault occurrence and clearing. In other words, by evaluating the reachable sets of hybrid automaton $H$, it can be examined at any onset in operations whether the system voltage settles down to unacceptable levels as time passes. The reachability analysis thus makes it possible to predict the voltage instability.

\section{Remarks}

Modeling of power system dynamics via hybrid automata is not a new approach. Hiskens et al. [5], [6] use a hybrid automaton for modeling of power systems with considering transformer tap positions and relay internal states. Fourlas et al. [18] also adopt a hybrid input/output automaton for an analysis of power transmission system. The previous works make it possible to formulate hybrid voltage dynamics in this paper.
Transient voltage instability as well as static instability has been studied by several researchers. Static voltage instability is a well-established subject of power engineering: see [1], [2] and references therein. For transient voltage instability, as defined in [1], the problem is concerned with a power system's ability to reach an acceptable operating condition following an event disturbance. With a power system model, the problem is mathematically translated into that of determining a stability region of attractor that corresponds to an acceptable operating condition. Moreover, it is stated in Section I that the problem is concerned with an ability to maintain system voltages in acceptable levels during transient period. This aspect of voltage problem is formulated in [1] as the problem of determining the size and shape of stability region. There are some previous works [19], [20], [21] on the transient voltage instability via energy functions.

The estimation of transient voltage instability is possible by evaluating backward reachable sets of hybrid automata as power system models. The method proposed here uses a backward reachable set for unsafe set which represents dangerous levels of system voltages. The complementary set to the reachable set may include the stability region. Therefore, the proposed method evaluates the stability region indirectly. Moreover, the complementary set provides quantitative measure of the stability region. This makes it possible to evaluate the size and shape of stability region. Thus, the problem of transient voltage instability is solved with the present formulation, which is based on reachable sets of hybrid automata.

The proposed method has many advantages of estimating transient voltage instability. The present attention to unsafe operations offers effective solutions for the following questions: how long does it take for system voltages to decrease by $10 \%$ ?; and how much do system voltages decrease in 10 seconds? Such quantitative information of voltage instability is important for operations of power systems. Unfortunately, it cannot be obtained with the previous methods [19], [20], [21], because they cannot fully handle the hybrid nature of voltage dynamics. Detailed simulations in power system analyzers are also used for voltage stability analysis. They can consider the hybrid nature of system dynamics. However, it does not contribute to the prediction of transient voltage instability and also does not provide any effective information for synthesizing controllers that make it possible to avoid the unsafe operations.

\section{APPLICATION TO SINGLE MACHINE-LOAD BUS SYSTEM}

This section applies the proposed method to an analysis of single machine-load bus (SMLB) system in Fig. 1. The SMLB system is given by Venkarasbramanian et al. [20] and consists of a synchronous machine, a load bus, and ac transmission lines. The aim of this application is to investigate whether the bus voltage of load reaches unacceptable levels, depending on a relay operation and continuous dynamics of synchronous machine.

A control action of relay devices is introduced for the present analysis. Suppose that one transmission route with two lines is tripped at time $t_{\mathrm{c}}(>0 \mathrm{~s})$ by relay devices. Fig. 1 also shows the control action and describes the change of transmission routes at $t=t_{\mathrm{c}}$. The control action generates two modes of operations shown in Fig. 1: (a) two routes 


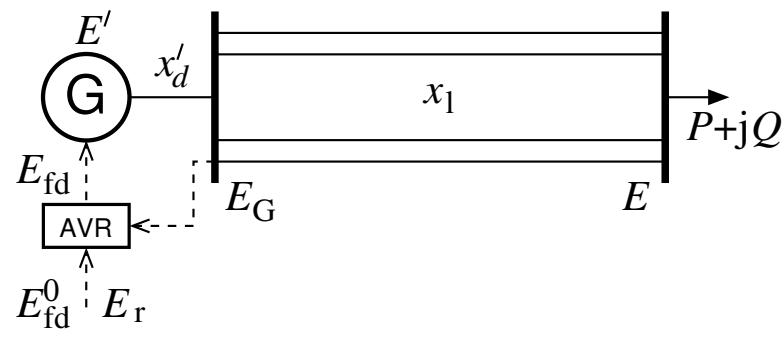

(a) two routes operation

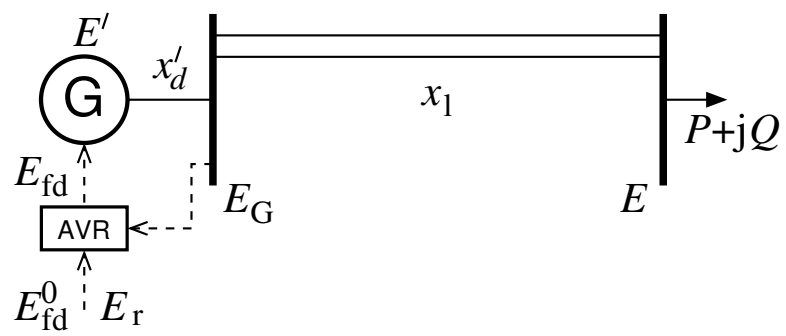

(b) one route operation

Fig. 1. Single machine-load bus (SMLB) system and control action following hybrid voltage dynamics. The SMLB system is given by Venkarasbramanian et al. [20] and consists of a synchronous machine, a load bus, and ac transmission lines. One transmission route with two lines is tripped at time $t_{\mathrm{c}}(>0 \mathrm{~s})$ by relay devices. Section. III aims at $0 \mathrm{~s}$ to predict the instability of bus voltage $E$ with considering the relay operation.

operation and (b) one route operation. The onset time $t_{\mathrm{c}}$ is a control parameter of the SMLB system. This section aims to predict the voltage instability governed by the hybrid dynamics at $0 \mathrm{~s}$.

\section{A. Modeling of continuous dynamics [20]}

Continuous voltage dynamics at each mode are modeled via a differential-algebraic equation (DAE). Suppose that the active power output of generator is equal to the consumed active power of load, and that there are no electro-mechanical dynamics of synchronous machine, i.e., the generator speed derivation is zero. Voltage dynamics are then isolated from the electro-mechanical dynamics. With a simplified one axis generator model and a first-order model of automatic voltage regulator (AVR), Venkarasbramanian et al. derive the following DAE for the voltage instability analysis:

$$
\left\{\begin{aligned}
T_{d 0}^{\prime} \dot{E}^{\prime}= & -\frac{x_{1}+x_{d}}{x^{\prime}} E^{\prime}+\frac{x_{d}-x_{d}^{\prime}}{x^{\prime}} \frac{E^{2}+x^{\prime} Q(E)}{E^{\prime}} \\
& +E_{\mathrm{fd}}, \\
\triangleq & f_{1}\left(E^{\prime}, E_{\mathrm{fd}}, E ; x_{1}\right), \\
T \dot{E}_{\mathrm{fd}}= & -\left(E_{\mathrm{fd}}-E_{\mathrm{fd}}^{0}\right)-K\left\{E_{\mathrm{G}}\left(E ; x_{1}\right)-E_{\mathrm{r}}\right\}, \\
\triangleq & f_{2}\left(E_{\mathrm{fd}}, E ; x_{1}\right), \\
= & E^{\prime 2} E^{2}-\left(x^{\prime} P\right)^{2}-\left\{x^{\prime} Q(E)+E^{2}\right\}^{2}, \\
\triangleq & g\left(E^{\prime}, E ; x_{1}\right),
\end{aligned}\right.
$$

TABLE I

PHYSICAL MEANING OF VARIABLES AND PARAMETERS FOR THE SMLB SYSTEM. THE PARAMETER SETTING EXCEPT $t_{\mathrm{C}}$ AND $E_{\mathrm{C}}$ IS BASED ON VENKARASBRAMANIAN et al. [20].

\begin{tabular}{lll}
\hline generator voltage behind transient reactance & $E^{\prime}$ \\
\hline field excitation & $E_{\mathrm{fd}}$ \\
\hline bus voltage of load & $E$ \\
\hline generator bus voltage & $E_{\mathrm{G}}$ & \\
\hline open-circuit transient time constant & $T_{d 0}^{\prime}$ & $5 \mathrm{~s}$ \\
\hline transmission reactance (two routes) & $x_{1}$ & 0.1 \\
\hline transmission reactance (one route) & $x_{1}$ & 0.2 \\
\hline$d$-axis synchronous reactance & $x_{d}$ & 1.2 \\
\hline$d$-axis transient reactance & $x_{d}^{\prime}$ & 0.2 \\
\hline time constant of first-order model of AVR & $T$ & $1 \mathrm{~s}$ \\
\hline nominal field excitation & $E_{\mathrm{fd}}^{0}$ & 2 \\
\hline gain constant of first-order model of AVR & $K$ & 7 \\
\hline set-point value of generator bus voltage & $E_{\mathrm{r}}$ & 1 \\
\hline mechanical input power to generator & $P_{\mathrm{m}}$ & 0.9 \\
\hline constant reactive power of load & $Q_{0}$ & $0.5 P_{\mathrm{m}}$ \\
\hline current source of load & $H$ & 0 \\
\hline impedance load & $B$ & 0 \\
\hline onset time of relay operation & $t_{\mathrm{c}}$ & $2.5 \mathrm{~s}$ \\
\hline critical value of bus voltage & $E_{\mathrm{c}}$ & 0.7 \\
\hline & & \\
\hline
\end{tabular}

where

$$
\begin{cases}E_{\mathrm{G}}\left(E ; x_{1}\right) & =\frac{1}{E} \sqrt{\left(x_{1} P\right)^{2}+\left\{x_{1} Q(E)+E^{2}\right\}^{2}}, \\ x^{\prime} & =x_{1}+x_{d}^{\prime}, \\ P & =P_{\mathrm{m}}, \\ Q(E) & =Q_{0}+H E+B E^{2} .\end{cases}
$$

The physical meaning of variables and parameters are shown in Tab. I. The load model also includes an algebraic dependence of reactive load on voltage. The reactive power $Q$ consists of a constant power source $Q_{0}$, a current source $H E$, and an impedance load $B E^{2}$.

Some mathematical structures of the DAE (2) are now reviewed. The subsets $L$ and $S$ in three-dimensional space $\left(E^{\prime}, E_{\mathrm{fd}}, E\right) \in \mathbb{R}^{3}$ are defined for the DAE (2) as

$$
\left\{\begin{aligned}
L\left(x_{1}\right) \triangleq & \left\{\left(E^{\prime}, E_{\mathrm{fd}}, E\right) \in \mathbb{R}^{3} \mid g\left(E^{\prime}, E ; x_{1}\right)=0\right\}, \\
S\left(x_{1}\right) \triangleq & \left\{\left(E^{\prime}, E_{\mathrm{fd}}, E\right) \in L\left(x_{1}\right)\right. \\
& \left.\mid \frac{\partial g}{\partial E}\left(E^{\prime}, E ; x_{1}\right)=0\right\} .
\end{aligned}\right.
$$

All solutions of the DAE (2) exist on the constraint set $L$. $L$ typically consists of a two-dimensional manifold in the threedimensional space. $S$ is called singular surface in which the solutions of the DAE (2) do not generally hold uniqueness properties. The DAE (2) describes a unique vector field on the subset or smooth manifold $L \backslash S$ in $\mathbb{R}^{3}$.

\section{B. Modeling of transition relation}

External jumps in the DAE (2) is central to modeling of transition relation which represents the route trip. The above subsection regards the switching of transmission routes as a control action. The switching is mathematically represented by a discontinuous change of parameter $x_{1}$ in the DAE (2). The values of $x_{1}$ are denoted as follows: $x_{1}^{-}$for two routes operation and $x_{1}^{+}$for one route operation. Then, the constraint set $L\left(x_{1}^{-}\right)$for two routes operation is different 


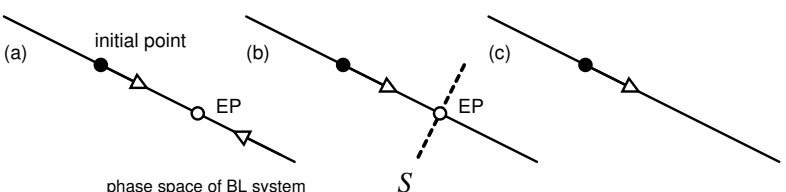

Fig. 2. External jumps and possible phase portraits in boundary layer (BL) system (5). The initial point is at $E=E\left(t_{\mathrm{c}}^{-}\right)$. The equilibrium point (EP) possibly coincides with $E=E\left(t_{\mathrm{c}}^{+}\right)$. The phase portraits, including stability of the EP, are parameterized by the values of $E^{\prime}\left(t_{\mathrm{c}}^{-}\right)$and $x_{1}^{+}$. In Fig. (a) the transition relation $e$ is modeled with the trajectory of the BL system (5). In Figs. (b) and (c) any transition cannot be defined in the present formalism.

from $L\left(x_{1}^{+}\right)$for one route operation. The difference is the origin of the transition $e$ at $t=t_{\mathrm{c}}$. Such behavior has been discussed for power system models [22], [23], [24] and is called external jumps [23]. The previous works [22], [23] characterize the external jumps via boundary layer (BL) systems.

This transition is discussed using phase portraits of a BL system. Now introduce the following remark presented in [22], [23]:

Remark If the DAE (2) admits of the external jump at $t=t_{\mathrm{c}}$, then the trajectory of the BL system

$$
\dot{E}=g\left(E^{\prime}\left(t_{\mathrm{c}}^{-}\right), E ; x_{1}^{+}\right),
$$

with the initial point $E=E\left(t_{\mathrm{c}}^{-}\right)$converges to the point $E\left(t_{\mathrm{c}}^{+}\right)$as time passes, satisfying $\left(E^{\prime}\left(t_{\mathrm{c}}^{-}\right), E_{\mathrm{fd}}\left(t_{\mathrm{c}}^{-}\right)\right)=$ $\left(E^{\prime}\left(t_{\mathrm{c}}^{+}\right), E_{\mathrm{fd}}\left(t_{\mathrm{c}}^{+}\right)\right)$.

This remark shows that the point $E=E\left(t_{\mathrm{c}}^{-}\right)$is on a stable manifold of the equilibrium point (EP) $E\left(t_{\mathrm{c}}^{+}\right)$in the $\mathrm{BL}$ system (5). Therefore, phase portraits in the BL system (5) play an important role in characterizing the external jumps. Fig. 2 shows examples of phase portraits in the BL system (5). Each of them is on one dimensional space. The initial point denotes $E=E\left(t_{\mathrm{c}}^{-}\right)$, and the EP possibly coincides with $E=E\left(t_{\mathrm{c}}^{+}\right)$. The three portraits in Fig. 2 are parameterized by the value of $E^{\prime}\left(t_{\mathrm{c}}^{-}\right)$and are explained as follows:

(a) The initial point exists on a stable manifold of the EP. In other words, the trajectory from the initial point converges to the EP as time passes. The EP exactly coincides with $E\left(t_{\mathrm{c}}^{+}\right)$;

(b) This denotes a critical phase portrait in which the EP exists on the singular surface $S$. The EP has zero eigenvalue, and the portrait is structurally unstable;

(c) The initial point does not exist on any stable manifold. Then, depending on detailed structures of the phase portrait, the trajectory from the initial point has a possibility of not converging to any steady state.

The transition relation $e$ at $t=t_{\mathrm{c}}$ is modeled via trajectories of the BL system (5). In Fig. 2(a), the transition $e$ is represented as the continuous trajectory that connects the initial point and the EP. In Figs. 2(b) and (c), any transition cannot be defined in the present formalism. This shows a loss of causality [25], [26] of the DAE (2) and possibly indicates a modeling breakdown. ${ }^{1}$ Here, to use the above modeling, it is

\footnotetext{
${ }^{1}$ It has been unclear whether the lose of model causality directly implies the system instability or not. This should be clarified by comparing numerical results of reduced power system models including the DAE (2) and detailed ones in power system simulators.
}

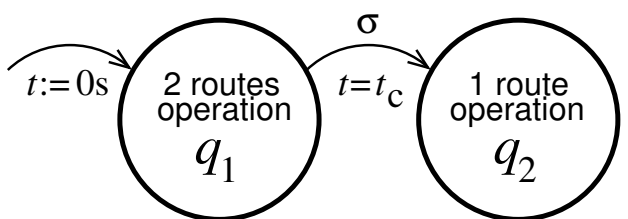

Fig. 3. Hybrid automaton (6) including one control action which represents the route trip

necessary to check at each transition whether the BL system (5) has the hyperbolic EP which stable manifold contains the initial point $E=E\left(t_{\mathrm{c}}^{-}\right)$. It is shown in [23] that the check is analytically possible for the BL system (5) with an energy function. Unfortunately, there is no analytical method for general DAE and BL systems. The check therefore relies on numerical simulations shown in Section III-D.

\section{Description of hybrid automaton and unsafe set}

With the hybrid automaton (1), the intersection of continuous voltage dynamics and relay operation in the SMLB system is represented as

$$
\left\{\begin{aligned}
& Q \cup X=\left\{q_{1}, q_{2}\right\} \cup\left(\mathbb{R}^{3} \times \mathbb{R}^{+}\right), \\
&\left(E^{\prime}, E_{\mathrm{fd}}, E, z\right) \in X, \\
& \mathcal{U} \cup \mathcal{D}= \emptyset, \\
& \Sigma_{u} \cup \Sigma_{d}=\{\sigma\} \cup \emptyset, \\
& f\left(q,\left(E^{\prime}, E_{\mathrm{fd}}, E, z\right)^{\mathrm{T}}\right), \bigcup_{i=1}^{2}\left(q_{i},\left(L_{i} \backslash S_{i}\right) \times \mathbb{R}^{+}\right), \\
& \operatorname{Dom}=\left(q,\left(E^{\prime}, E_{\mathrm{fd}}, E, z\right)^{\mathrm{T}}, \sigma\right),
\end{aligned}\right.
$$

where $L_{1}=L\left(x_{1}^{-}\right), L_{2}=L\left(x_{1}^{+}\right), S_{1}=S\left(x_{1}^{-}\right)$, and $S_{2}=$ $S\left(x_{1}^{+}\right)$. Fig. 3 describes the hybrid automaton (6) with two modes and one control action. The continuous state space is augmented with a timer $z \in \mathbb{R}^{+}$in order to force the transition relation $e$. The discrete variable $q_{1}$ is assigned to the two routes operation and $q_{2}$ to the one route operation. The relay operation is also regarded as the control action $\sigma$. The continuous vector field $f$ on Dom is represented as

$$
\begin{gathered}
f\left(q,\left(E^{\prime}, E_{\mathrm{fd}}, E, z\right)^{\mathrm{T}}\right)= \\
\frac{1}{T_{d 0}^{\prime}} f_{1}\left(E^{\prime}, E_{\mathrm{fd}}, E ; x_{1}\right) \\
\frac{1}{T} f_{2}\left(E_{\mathrm{fd}}, E ; x_{1}\right) \\
-\left\{\frac{\partial g}{\partial E}\left(E^{\prime}, E ; x_{1}\right)\right\}^{-1} \cdot \frac{2 E^{\prime} E^{2}}{T_{d 0}^{\prime}} f_{1}\left(E^{\prime}, E_{\mathrm{fd}}, E ; x_{1}\right) \\
1 \quad \begin{array}{ll}
x_{1}=x_{1}^{-} & \text {if } q=q_{1}, \\
x_{1}=x_{1}^{+} & \text {if } q=q_{2} .
\end{array}
\end{gathered}
$$

The present description of vector field is given in [20]. The vector field has the first integral $g\left(E^{\prime}, E ; x_{1}\right)$, and its trajectories therefore satisfy the algebraic constraint $g\left(E^{\prime}, E ; x_{1}\right)$ 
in the DAE (2). Thus, with the description of vector field, the trajectories of the DAE (2) are derived. The transition relation $e$ is driven by the control action $\sigma$ and is given by the trajectories of the BL system (5). Suppose that $\phi_{t}\left(\cdot ; E^{\prime}\left(t_{\mathrm{c}}^{-}\right), x_{1}^{+}\right)$is a flow defined by the BL system (5), then the transition $e$ is represented by

$$
\begin{aligned}
& e\left(q_{1},\left(E^{\prime}\left(t_{\mathrm{c}}^{-}\right), E_{\mathrm{fd}}\left(t_{\mathrm{c}}^{-}\right), E\left(t_{\mathrm{c}}^{-}\right), t_{\mathrm{c}}\right)^{\mathrm{T}}, \sigma\right) \\
& =\left(q_{2},\left(E^{\prime}\left(t_{\mathrm{c}}^{+}\right), E_{\mathrm{fd}}\left(t_{\mathrm{c}}^{+}\right), E\left(t_{\mathrm{c}}^{+}\right), t_{\mathrm{c}}\right)^{\mathrm{T}}\right),
\end{aligned}
$$

where

$$
\left\{\begin{array}{l}
E^{\prime}\left(t_{\mathrm{c}}^{+}\right)=E^{\prime}\left(t_{\mathrm{c}}^{-}\right), \\
E_{\mathrm{fd}}\left(t_{\mathrm{c}}^{+}\right)=E_{\mathrm{fd}}\left(t_{\mathrm{c}}^{-}\right), \\
E\left(t_{\mathrm{c}}^{+}\right)=\lim _{t \rightarrow+\infty} \phi_{t}\left(E\left(t_{\mathrm{c}}^{-}\right) ; E^{\prime}\left(t_{\mathrm{c}}^{-}\right), x_{1}^{+}\right) .
\end{array}\right.
$$

Under the parameter setting in Tab. I, the above representation of the transition $e$ is valid only if the value $E\left(t_{\mathrm{c}}^{+}\right)$is bounded. If otherwise, any transition cannot be described in the present formalism. In numerical simulations of Section III-D, if the above situation of transition occurs, then the numerical simulations are stopped.

The proposed method in Section II requires detailed information about unacceptable operations of the SMLB system and associated unsafe sets of the hybrid automata (6). The present analysis is performed under the following unsafe set $G$ and the set Init of initial conditions:

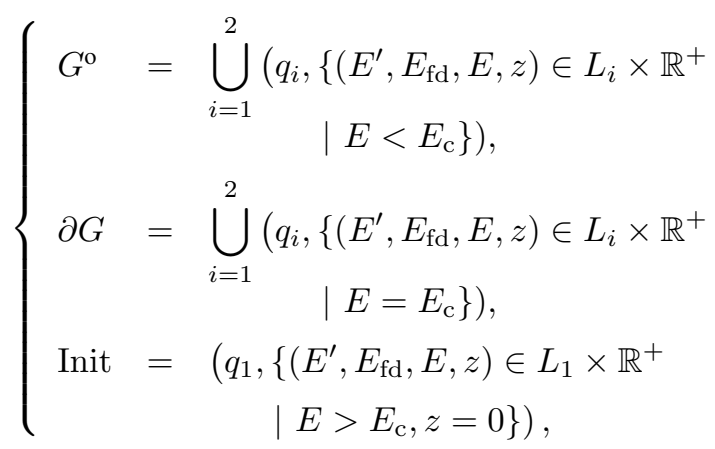

where $E_{\mathrm{c}}=0.7$. All states in $G$ physically imply unacceptable operations of the SMLB system because of the decrease of bus voltage of load.

The definition of unsafe sets is crucial for the proposed method in this paper. The prediction of voltage instability strongly depends on how unacceptable states of a power system are set. The application here aims to predict the instability of bus voltage $E$, depending on the generator dynamics and the route trip. The above setting of $E_{\mathrm{c}}$ is meaningful for preventing its very low value. Here, the DAE possesses a singular surface $S$ as a subset of $L$ satisfying $E<0.7$. Namely, the surface $S$ is contained in the unsafe set $G^{\mathrm{o}}$.

\section{Numerical reachable set and prediction}

This section shows a numerical result of reachable set in the hybrid automaton (6). The parameter setting, given in Tab. I, is based on Venkarasbramanian et al. [20] except $t_{\mathrm{c}}$ and $E_{\mathrm{c}}$. The computation of reachable set in each individual mode is straightforward and is accomplished by computing time-reverse trajectories from usable parts [27] on the boundary $\partial G$. This paper adopts the 3rd-stage Radau-IIA implicit Runge-Kutta method [28] for numerical integration.

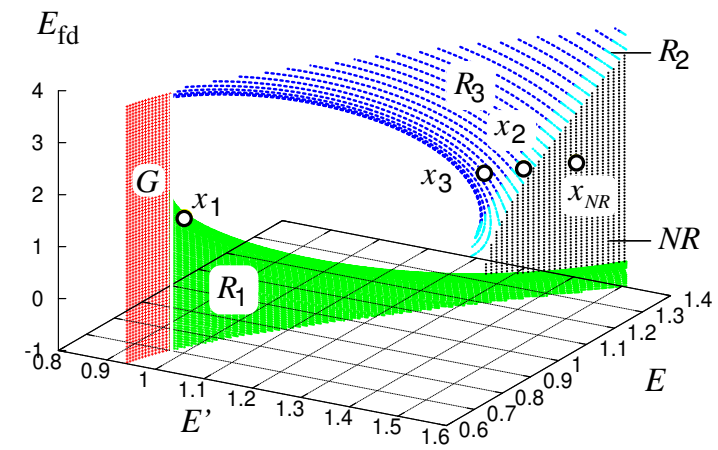

Fig. 4. Reachable set of the hybrid automaton (6). Init is the two dimensional plane in the space $\left(E^{\prime}, E_{\mathrm{fd}}, E\right) \in \mathbb{R}^{3}$ and corresponds to the plane containing the sets $R_{i}(i=1,2,3)$ and $N R$. The union of the sets $R_{i}$ is the reachable set.

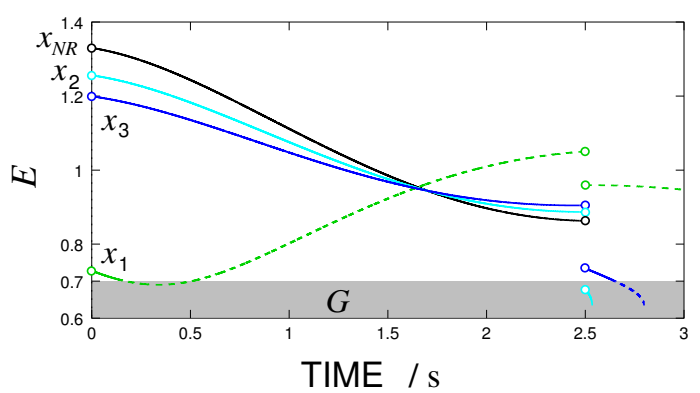

Fig. 5. Transient behavior from four initial conditions in Fig. 4. All the behavior reaches the unsafe set and is characterized by the reachable set in Fig. 4.

The problem here is how to deal with the interaction between the two modes. For the present case, the following three reachable set computations are necessary:

(a) The set of states which lead to voltage instability in the mode $q_{1}$. This set is obtained as time-reverse trajectories from the usable parts in time $0 \mathrm{~s}$ to $-t_{\mathrm{c}}$ at the mode $q_{1}$

(b) The set of states which lead to voltage instability in the mode $q_{2}$. This set is also given by time-reverse trajectories from the usable parts in time $0 \mathrm{~s}$ to $-\infty$ at the mode $q_{2}$

(c) The set of states which, after mapped from the mode $q_{1}$, lead to voltage instability in the mode $q_{2}$. This set is derived as follows: First, the set of states at the mode $q_{1}$ is computed which are mapped by the transition $e$ onto the derived reachable set in (b). Second, the timereverse trajectories from the obtained set are computed during the interval $\left[0 \mathrm{~s},-t_{\mathrm{c}}\right]$. Lastly, all the states on the trajectories at time $-t_{\mathrm{c}}$ form our questing reachable set.

Figure 4 shows the reachable set of the hybrid automaton (6). The figure shows the set Init of initial conditions and the unsafe set $G$. Init is the two dimensional plane in the space $\left(E^{\prime}, E_{\mathrm{fd}}, E\right) \in \mathbb{R}^{3}$ and corresponds to the plane containing the sets $R_{i}(i=1,2,3)$ and $N R$ in Fig. 4. The union of the sets $R_{i}$ is the reachable set. $R_{1}$ is the subset of Init from 
which any trajectory reaches $\partial G$ before the transition $e . R_{2}$ is the subset of Init from which any trajectory directly lands on $G$ by the transition $e . R_{3}$ is also the subset of Init from which any trajectory reaches $\partial G$ after the transition $e$. The region $N R$ is the subset of Init in which any transition cannot be defined, i.e., the case (c) of phase portraits in Fig. 2. The white region in Init of Fig. 4 therefore corresponds to the transient voltage stability region with considering the transition relation $e$. Fig. 5 shows the transient behavior starting from the four initial conditions in Fig. 4. All the behavior in Fig. 5 reaches the unsafe set and is characterized by the reachable set. The figures show that the fate of bus voltage $E$ can be estimated at $0 \mathrm{~s}$. The prediction of transient voltage instability is hence achieved via the computation of reachable sets.

\section{CONCLUDING REMARKS}

This paper proposed to use reachable sets of hybrid automata for the prediction of transient voltage instability. The proposed method is based on modeling of voltage dynamics via hybrid automata which include nonlinear DAEs. Linearized discrete time models are used in [8], [9], [10] for analysis of voltage behavior. The nonlinear nature of the modeling makes it possible to examine global voltage dynamics of power systems, in other words, voltage behavior far from acceptable operating conditions.

The proposed method is also based on reachability analysis of hybrid automata. Computation of reachable sets is crucial for the prediction of voltage instability. The present computation for the SMLB system is simple. It is therefore non-trivial whether its computation strategy in Section III is generalized into the cases of complicated transition relations and high-dimensional DAEs. Non-uniqueness in reverse time of trajectories [29] also becomes a limitation of the computation. An effective computation for DAE-based models is a next challenging issue for analysis of actual bulk power systems.

\section{ACKNOWLEDGMENTS}

The first author is grateful to Mr. Hiroaki Ebina and Mr. Yusuke Satake for valuable discussions and numerical simulations. He is also grateful to Professor Ian Mitchell for suggestive advises on reachable set computation. The authors are also grateful to anonymous reviewers for their valuable and critical comments.

\section{REFERENCES}

[1] H. D. Chiang, "Power system stability," in Wiley Encyclopedia of Electrical and Electronics Engineering, J. G. Webster, Ed. New York: John Wiley \& Sons, March 1999, pp. 105-137.

[2] NERC Report, Survey of the Voltage Collapse Phenomena, Summary of the Interconnection Dynamics Task Force's Survey on the Voltage Collapse Phenomena, August 1991.

[3] S. Larsson and E. Ek, "The black-out in southern Sweden and eastern Denmark, September 23, 2003," in Proceedings of the IEEE PES General Meeting, vol. 2, Denver, USA, 2004, pp. 1688-1672.

[4] M. Domenica, D. Benedetto, and A. Sangiovanni-Vincentelli, Eds., Hybrid Systems: Computation and Control, ser. Lecture Notes in Computer Science 2034. Springer-Verlag, 2001.

[5] I. A. Hiskens and M. A. Pai, "Hybrid systems view of power system modeling," in Proceedings of the 2000 International Symposium on Circuits and Systems, vol. II, Geneva, Switzerland, 2000, pp. 228231.

[6] I. A. Hiskens, "Power system modeling for inverse problems," IEEE Transactions on Circuits and Systems-I: Fundamental Theory and Applications, vol. 51, no. 3, pp. 539-551, March 2004.
[7] Q. Y. Tong, G. F. Yan, and G. Z. Zhao, "Hybrid system view of voltage instability problem," in Proceedings of the Second International Conference on Machine Learning and Cybernetics, X'ian, China, November 2003, pp. 915-918.

[8] T. Geyer, M. Larsson, and M. Morari, "Hybrid emergency voltage control in power systems," in Proceedings of the European Control Conference 2003, Cambridge, UK, September 2003.

[9] A. Giovanni, T. Geyer, and M. Morari, "A hybrid system approach to power systems voltage control," in Proceedings of the 44th Conference on Decision and Control, and the European Control Conference 2005, Seville, Spain, December 2005, pp. 6774-6779.

[10] S. Leirens, J. Buisson, P. Bastard, and J. L. Coullon, "A hybrid approach for voltage stability of power systems," in Proceedings of the 15th Power Systems Computation Conference, Liege, Belguim, August 2005.

[11] T. Hikihara, "Application of hybrid system theory to power system analysis (I)," in Annual Meeting Record I.E.E.Japan, vol. 6, 2005, p. 187, (in Japanese).

[12] Y. Susuki, H. Ebina, and T. Hikihara, "Application of hybrid system theory to power system stability analysis," in Proceedings of the 2005 International Symposium on Nonlinear Theory and its Applications, Bruge, Belgium, October 18-21 2005, pp. 202-205.

[13] T. A. Henzinger, "The theory of hybrid automata," in Proceedings of the 11th Annual IEEE Symposium on Logic in Computer Science, 1996, pp. 278-292.

[14] C. J. Tomlin, I. Mitchell, A. M. Bayen, and M. Oishi, "Computational techniques for the verification of hybrid systems," Proceedings of the IEEE, vol. 91, no. 7, pp. 986-1001, July 2003.

[15] Y. Susuki, H. Ebina, and T. Hikihara, "Predicting transient instability of power systems based on hybrid system reachability analysis," in Preprints of the IFAC Symposium on Power Plants and Power Systems Control, Kananaskis, Canada, June 25-28 2006.

[16] K. R. Padiyar, HVDC Power Transmission Systems: Technology and System Interactions. New Delhi: Wiley Eastern, 1990.

[17] CIGRÉ and IEEE FACTS Working Groups. FACTS Overview, IEEE Catalog \#95-TP-108, 1995.

[18] G. K. Fourlas, K. J. Kyriakopoulos, and C. D. Vournas, "Hybrid systems modeling for power systems," IEEE Circuits and Systems Magazine, vol. 4, no. 3, pp. 16-23, Third Quarter 2004.

[19] C. C. Liu and K. T. Vu, "Analysis of mechanisms of voltage instability in electric power systems," in Systems and Control Theory for Power Systems, J. Chow, P. V. Kokotovic, and R. J. Thomas, Eds. New York: Springer-Verlag, 1995, vol. 64, pp. 235-258.

[20] V. Venkatasubramanian, H. Schättler, and J. Zaborszky, "Voltage dynamics: Study of a generator with voltage control, transmission, and matched MW load," IEEE Transactions on Automatic Control, vol. 37, no. 11, pp. 1717-1733, November 1992.

[21] K. L. Praprost and K. A. Loparo, "An energy function method for determining voltage collapse during a power system transient," IEEE Transactions on Circuits and Systems-I: Fundamental Theory and Applications, vol. 41, no. 8, pp. 635-651, October 1994.

[22] S. Sastry and P. Varaiya, "Hierarchical stability and alert steering control of interconnected power systems," IEEE Transactions on Circuits and Systems, vol. CAS-27, no. 11, pp. 1102-1112, November 1980.

[23] C. C. Chu, "Transient dynamics of electric power systems: Direct stability assessment and chaotic motions," PhD Dissertation, Cornell University, January 1996.

[24] Y. Zou, M. H. Yin, and H. D. Chiang, "Theoretical foundation of the controlling UEP method for direct transient-stability analysis of network-preserving power system models," IEEE Transactions of Circuits and Systems-I: Fundamental Theory and Applications, vol. 50, no. 10, pp. 1324-1336, Octorber 2003.

[25] C. L. DeMarco and A. R. Bergen, "Application of singular perturbation techniques to power system transient stability analysis," in Proceedings of the International Symposium on Circuits and Systems, 1984, pp. 597-601.

[26] H. G. Kwatny, A. K. Pasrija, and L. Y. Bahar, "Static bifurcations in electric power networks: Loss of steady-state stability and voltage collapse," IEEE Transactions on Circuits and Systems, vol. CAS-33, no. 10, pp. 981-991, Octorber 1986

[27] T. Başar and G. J. Olsder, Dynamic Noncooperative Game Theory. Philadelphia: SIAM, 1998.

[28] E. Hairer and G. Wanner, Solving Ordinary Differential Equations 2. Stiff and Differential-Algebraic Problems, 2nd ed., ser. Springer Series in Computational Mathematics. Berlin Heidelberg: Springer-Verlag, 1996, vol. 14

[29] I. A. Hiskens, "Non-uniqueness in reverse time of hybrid system trajectories," in Hybrid Systems: Computation and Control, ser. Lecture Notes in Computer Science 3414, M. Morari and L. Thiele, Eds. Springer-Verlag, 2005, pp. 339-353. 